\title{
Delayed Airway Obstruction in Posterior Fossa Craniotomy with Park-Bench Position-A Case Report and Review of the Literatures
}

\author{
Szu-Kai Hsu, Cheng-Ta Hsieh, Chih-Ta Huang, Jing-Shan Huang \\ Division of Neurosurgery, Department of Surgery, Cathay General Hospital, Taipei, Taiwan \\ Email: sky.hsu@gmail.com
}

Received September 3, 2012; revised October 1, 2012; accepted October 11, 2012

\begin{abstract}
Background: Park-bench position is a commonly used position in patients operated with cerebellopontine tumor, microvascular decompression, or lesions of cerebellar hemisphere and vertebral artery. Several complications associated with this positioning have been described. However, the delayed airway complication resulting from park-bench position has been rarely reported in the literatures. Herein, we report a rare case of delayed airway obstruction after a posterior-fossa craniotomy with park-bench position for acoustic neuroma. Case Presentation: A 43-year-old woman, who has a right acoustic neuroma, was placed in the park-bench position for a posterior-fossa craniotomy. The anesthesia and operation were performed smoothly. However, progressive swelling in the left neck with extending to left face occurred two hours after endo-tracheal extubation. Computed tomographic scan of neck revealed diffuse swelling of the muscles and deep soft tissue in the left side. No obvious embolization of vascular system including jugular vein was found in the sonography. Because of obstruction in upper airway, orotracheal intubation and a head-up position were performed. The clinical symptoms improved gradually since the third post-operative day. She was discharged with obvious complications on the 23rd day after the operation. Conclusion: The possible mechanisms may originate from the kinking of the jugular vein due to hyper-flexed neck position during surgery. To prevent such complication, we must take great care of the anesthetized patients when placed in the forced neck position and always keep this rare but so critical complication in mind.
\end{abstract}

Keywords: Craniotomy; Intraoperative Position; Neck Swelling; Park-Bench Position

\section{Introduction}

Park-bench position is a very common posture for posterior fossa craniotomy in management of patients with cerebellopontine tumor, lesions of cerebellar hemisphere or vascular diseases. The effects of gravity and mechanical force exerted on the neck and extremities frequently used to facilitate the neurological procedures when the park-bench position was placed. Several complications related to this position, including decubitus ulcer, air embolism, swelling of facial or neck, or damage to the cervical spinal cord, brachial plexus, and peripheral nerve, have been well-documented [1,2]. However, delayed airway obstruction related to park-bench position after posterior fossa surgery has been rarely reported in the literatures. Herein, we reported a case of delayed airway obstruction resulting from severe swelling of the neck and face after a posterior fossa surgery in the park bench position.

\section{Case Report}

A 46-year-old female patient complained with progres- sive right hearing impairment followed by unsteady gait one year before admission. At admission, neurological examinations revealed right hearing impairment and right-deviated unsteady gait. The laboratory examinations revealed no remarkable contribution. Magnetic resonance (MR) images showed a cerebellopontine tumor with $3 \mathrm{~cm}$ in diameter in the right posterior fossa, which enlarged inner auditory canal. Then she underwent a right sub-occipital craniotomy to remove the tumor. After endotracheal intubation with a 7-mm tube, a central venous catheter was inserted in the left femoral vein. The left park bench position with neck flexion and rotation was placed. The 2-fingerbreadth clearance between the chin and thorax was confirmed. During an eight-hour procedure, the tumor was totally resected. No obvious hemorrhage or obstruction of venous system was noted. At operation, the mean systolic blood pressure was 120 mmHg. The total amount of delivered fluid was $1700 \mathrm{~mL}$ and her urine output was $2900 \mathrm{~mL}$. No blood transfusion was done. Then she was managed in the intensive care unite. However, progressive swelling of the left neck and 
face resulting into dyspnea and stridor were found two hours after extubation (Figure 1(a)). The re-intubation was performed to protect the airway.

The computed tomographic (CT) scan of neck showed diffuse swelling of the tracheal mucosa, muscles and deep soft tissue and the area below the thyroid cartilage was spared (Figures 1(b) and (c)). The Doppler sonography of neck revealed the narrowing of left jugular vein without obvious embolization or obstruction. Conservative treatment with head-up and neutral position was performed. The swelling gradually subsided at the third day after surgery (Figure 2(a)) and returned to normal appearance on the seventeenth day after surgery (Figure 2(b)). The subsequent CT scan of the neck revealed improved swelling without compression of trachea (Figure 2(c)). After extubation on the seventeenth postoperative day, no obvious neurological deficits were found. She was discharged on the 23rd day after the surgery.

\section{Discussion}

Several complications related to park-bench position, including decubitus ulcer, air embolism, swelling of facial or neck, or damage to the cervical spinal cord, brachial plexus, and peripheral nerve, have been welldocumented $[1,2]$. The swelling of the neck after neurosurgical procedures has very rarely been reported in the field of anesthesiology and neurosurgery [2-9]. The majority of the affected individuals had undergone posterior fossa surgery in the sitting position [2]. The delayed airway obstruction resulting from the severe neck swelling in a park-bench position after a posterior fossa surgery is rarely described and only one similar case has been reported in the literatures [2] (Table 1).

Shimuzi et al. reported a case of neck swelling and brachial plexopathy as park-bench position-related complications. Erosion of the skin (severe compression) on the left side of the neck was observed. A CT scan of neck showed marked swelling with displacement of the trachea toward the right and thrombus formation in the right internal jugular vein. The kinking of the internal jugular vein due to excessive neck flexion has been mainly hypothesized as the major cause of disturbed venous drainage in the postoperative patients [3-5,7-8]. Most patients exhibited swelling for 2 to 3 weeks (form 1 day to 12 weeks) because it may be necessary that the venous re turn in the external jugular system must be restored via the development of a collateral venous pathway. Other infrequently causes including 1) angioedema, 2) vascular lesion, 3) infection, 4) denervation, 5) lymphedema and 6) postoperative acute sialadenitis also have been also reported to cause the neck swelling.

In our patient, no skin ecchymosis, blister, palpable

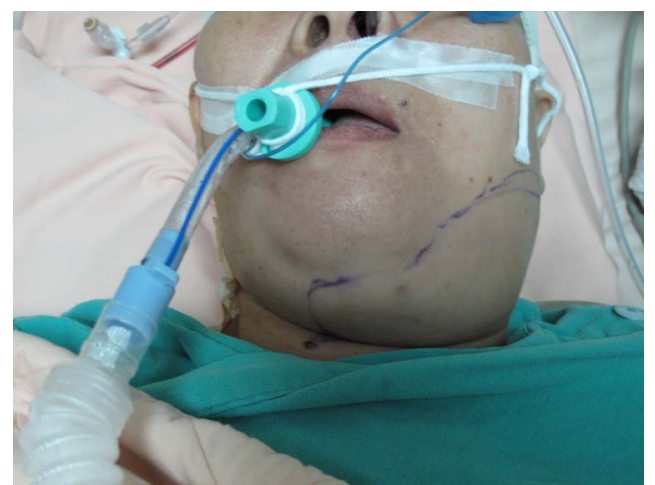

(a)

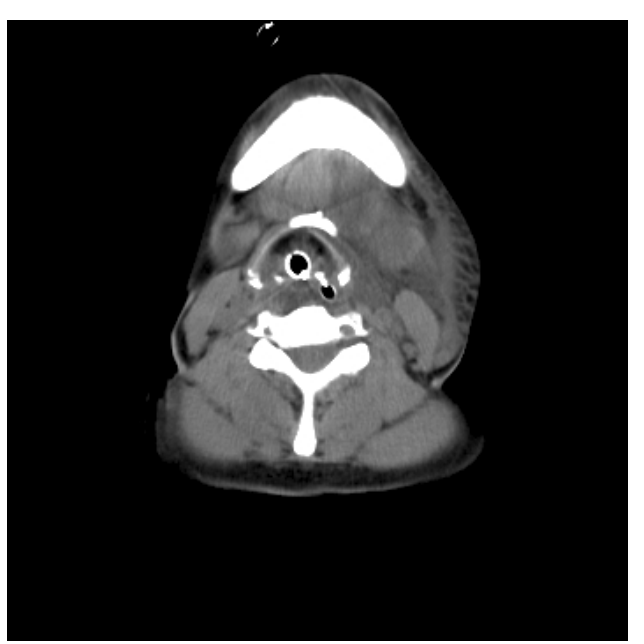

(b)

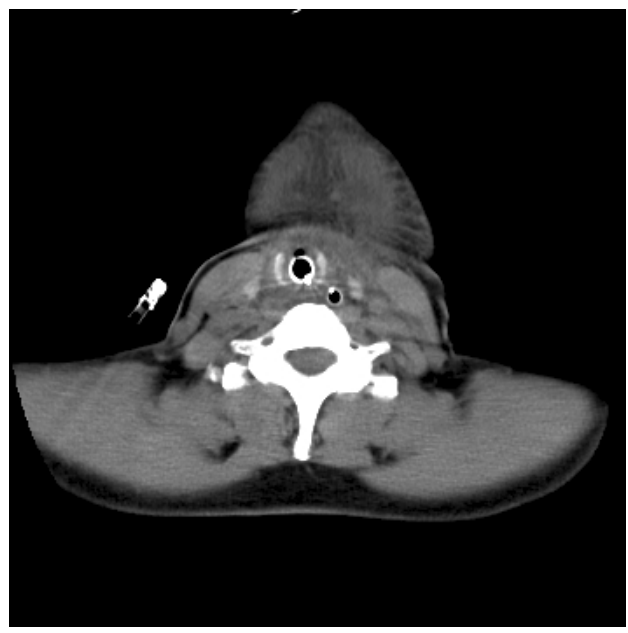

(c)

Figure 1. (a) Two hours after extubation, acute left neck and sub-mandibular area swelling with stridor and dyspnea was noted. Then, emergent re-intubation was performed immediately; (b) Computed tomography (CT) showed left neck diffuse swelling of the tracheal mucosa, muscles and deep soft tissue. The space between orotracheal tube and tracheal mucosa was almost disappeared (2 hours after extubation); (c) The area below the thyroid cartilage was spared (2 hours after extubation). 


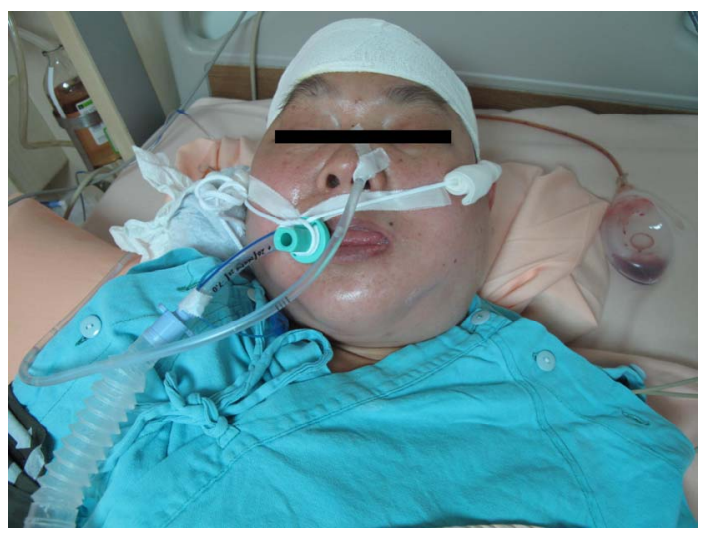

(a)

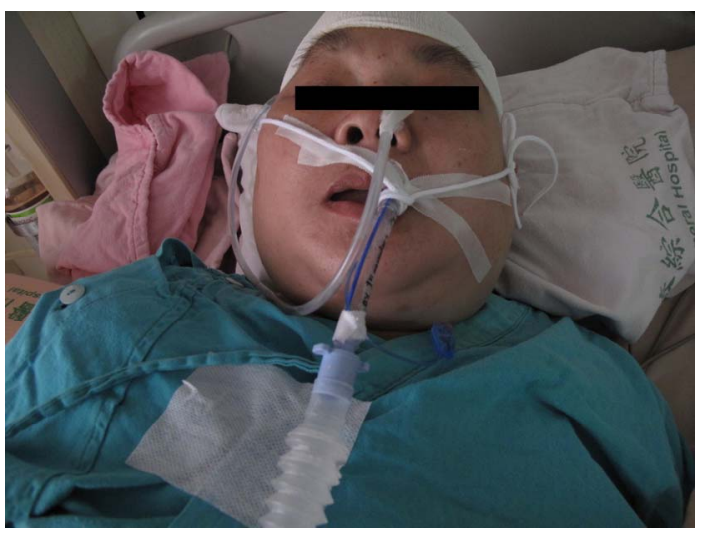

(b)

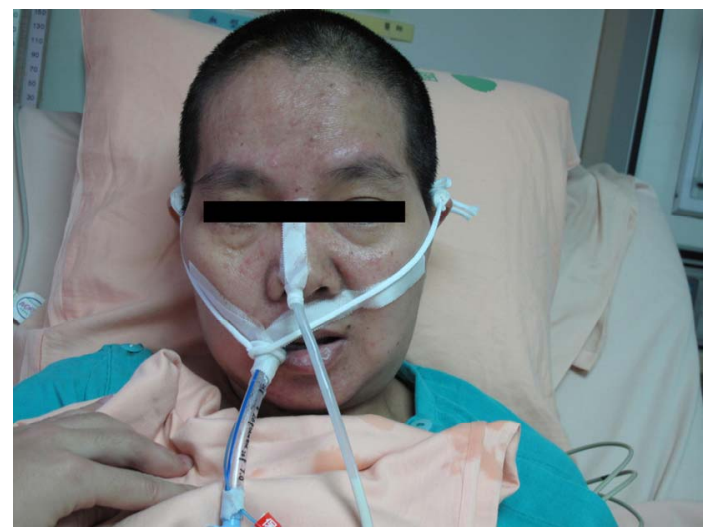

(c)

Figure 2. (a) The swelling progressed over the next 10 hours to involve the whole face and bilateral neck (12 hours after extubation); (b) On the 3rd postoperative day, the swelling was improving; (c) On the 17th postoperative day, the neck and facial swelling improved to normal status.

Table 1. The summary of two cases, including our patient, with the swelling of neck and face in a park-bench position.

\begin{tabular}{|c|c|c|c|c|c|c|c|c|c|}
\hline \multirow{2}{*}{ Authos } & \multirow{2}{*}{ Age/sex } & \multirow{2}{*}{$\begin{array}{l}\text { Surgery } \\
\text { position }\end{array}$} & \multirow{2}{*}{ Time (h) } & \multirow{2}{*}{$\begin{array}{l}\text { Brain } \\
\text { edema }\end{array}$} & \multirow{2}{*}{ Risk } & \multicolumn{4}{|c|}{ Postoperative swelling } \\
\hline & & & & & & region & onset & duration & outcome \\
\hline Shimizu S, 2009 & $56 / F$ & $\begin{array}{l}\text { Left park } \\
\text { bench }\end{array}$ & 10 & Nil & $\begin{array}{c}\text { Direct } \\
\text { compression }\end{array}$ & $\begin{array}{c}\text { Face and } \\
\text { neck }\end{array}$ & $2 \mathrm{~h}$ & $10 \mathrm{~d}$ & $\begin{array}{l}\text { Left brachial } \\
\text { plexopathy }\end{array}$ \\
\hline Present case & $43 / F$ & $\begin{array}{l}\text { Left park } \\
\text { bench }\end{array}$ & 8 & Nil & Nil & $\begin{array}{c}\text { Face and } \\
\text { neck }\end{array}$ & $2 \mathrm{~h}$ & $17 \mathrm{~d}$ & Good \\
\hline
\end{tabular}

mass or erosion was found at the neck area. Neither local heat nor tenderness was noted. The CT scan of neck showed no obvious hematoma. The disturbed venous or lymphatic drainage should be considered as the major mechanisms in our patient. The surgical position during prolonged operative time was more preferred to induce this devastating complication. Although no definite mechanism was detected, we thought the venous and lymph obstruction should play some roles in our case.

However, future investigations should address the changes in jugular venous anatomy, blood flow and neck lymph anatomy associated with positioning for posterior cranial fossa surgery and the mechanisms leading to the persistence/progression of edema in the postoperative period. In management of this devastating complication, early intubation or tracheostomy to protect airway was recommended $[5,8]$

\section{Conclusion}

Although the neck swelling resulting into airway compression in patients placed in bark-bench position for posterior fossa craniotomy is a rare but devastating complication, our case posed to the serious information about the anesthetized patients placing in the forced positions of the neck. If postoperative neck swelling with tracheal compression appears, airway protection with early intu- 
bation or tracheostomy is the most important.

\section{REFERENCES}

[1] F. W. M. Cheney, K. B. M. Domino, R. A. M. Caplan and K. L. P. Posner, "Nerve Injury Associated with Anesthesia: A Closed Claims Analysis,” Anesthesiology, Vol. 90, No. 4, 1999, pp. 1062-1069.

[2] S. Shimizu, K. Sato, I. Mabuchi, S. Utsuki, H. Oka and S. Kan, "Brachial Plexopathy Due to Massive Swelling of the Neck Associated with Craniotomy in the Park Bench Position,” Surgical Neurology, Vol. 71, No. 4, 2009, pp. 504-509.

[3] S. C. Ellis, C. W. Bryan-Brown and H. Hyderally, "Massive Swelling of the Head and Neck," Anesthesiology, Vol. 42, No. 1, 1975, pp. 102-103.

[4] J. M. D. Mayhew, M. M. D. Miner, J. M. B. Katz and B. Ch, "Macroglossia in a 16-Month-Old Child after a Craniotomy,” Anesthesiology, Vol. 62, No. 5, 1985, p. 683.
[5] R. M. D. McAllister, "Macroglossia-A Positional Complication,” Anesthesiology, Vol. 40, No. 2, 1974, p. 199.

[6] C. A. Munshi, M. S. Dhamee and G. Sk, "Postoperative Unilateral Facial Oedema: A Complication of Acute Flexion of the Neck," Canadian Anesthesiologists' Society, Vol. 31, No. 2, 1984, pp. 197-199.

[7] V. B. Narayan and G. S. Umamaheswara, "Unilateral Facial and Neck Swelling after Infratentorial Surgery in the Lateral Position,” Anesthesia and Analgesia, Vol. 89, No. 5, 1999, pp. 1290-1291.

[8] M. P. Tattersall, "Massive Swelling of the Face and Tongue. A Complication of Posterior Cranial Fossa Surgery in the Sitting Position,” Anaesthesia, Vol. 39, No. 10, 1984, pp. 1015-1017.

[9] E. Teeple and J. R. R. Maroon, "Hemimacroglossia and Unilateral Ischemic Necrosis of the Tongue in a LongDuration Neurosurgical Procedure," Anesthesiology, Vol. 64, 1986, pp. 845-846. 